\title{
Protective effect of Xingnaojia formulation on rats with brain and liver damage caused by chronic alcoholism
}

\author{
SHUANG LI ${ }^{1 *}$, SU WANG $^{2 *}$, ZHI-GANG GUO ${ }^{1^{*}}$, NING HUANG $^{3^{*}}$, FAN-RONG ZHAO ${ }^{3 *}$, MO-LI ZHU $^{3 *}$, \\ LI-JUAN MA M $^{3^{*}}$, JIN-YING LIANG ${ }^{3 *}$, YU-LIN ZHANG ${ }^{3^{*}}$, ZHONG-LIN HUANG $^{3^{*}}$ and GUANG-RUI WAN ${ }^{1}$ \\ ${ }^{1}$ School of Basic Medical Sciences; ${ }^{2}$ College of Pharmacy, The Third Affiliated Hospital; \\ ${ }^{3}$ College of Pharmacy, Xinxiang Medical University, Xinxiang, Henan 453003, P.R. China
}

Received May 15, 2014; Accepted July 31, 2015

DOI: $10.3892 /$ etm.2015.2773

\begin{abstract}
The aim of this study was to observe the effect of a formulation of traditional Chinese medicine extracts known as Xingnaojia (XNJ) on the liver function, learning ability and memory of rats with chronic alcoholism and to verify the mechanism by which it protects the brain and liver. A rat model of chronic alcoholism was used in the study. The spatial learning ability and memory of the rats were tested. The rats were then sacrificed and their brains and hepatic tissues were isolated. The activity of superoxide dismutase (SOD) and levels of glutamate (Glu), N-methyl D-aspartate receptor subtype 2B (NR2B), cyclin-dependent kinase 5 (CDK5) and cannabinoid receptor 1 (CB1) in the hippocampus were analyzed. The ultrastructure of the hepatic tissue was observed by electron microscopy. In addition, the activities of alcohol dehydrogenase (ADH) and aldehyde dehydrogenase (ALDH) in serum were tested and the levels of low-density lipoprotein (LDL), high-density lipoprotein (HDL), triglycerides (TG) and total cholesterol (TCHOL) were analyzed. XNJ enhanced the learning and memory of rats with chronic alcoholism. Treatment with XNJ increased the activity of SOD, and decreased the expression levels of NR2B mRNA and NR2B, $\mathrm{CB} 1$ and CDK5 proteins in the brain tissues compared with those in the model rats. It also increased the activity of ALDH in the serum and liver, decreased the serum levels of LDL, TG and TCHOL and increased the serum level of HDL. These results indicate that $\mathrm{XNJ}$ exhibited a protective effect against brain and liver damage in rats with chronic alcoholism.
\end{abstract}

Correspondence to: Professor Guang-Rui Wan, School of Basic Medical Sciences, Xinxiang Medical University, 601 Jinsui Road, Xinxiang, Henan 453003, P.R. China

E-mail: guangruiwancn@126.com

*Contributed equally

Key words: Xingnaojia, chronic alcoholism, cerebral injury, hepatic injury

\section{Introduction}

Alcoholism is a common, but serious worldwide social and medical problem. Reduction of lifespan due to alcoholism is more significant than that due to cardiovascular diseases. The combination of health, psychological and social problems associated with alcoholism and alcohol abuse is a major public health problem (1). Long term drinking of alcohol can cause numerous pathological changes in the body and is particularly harmful to the brain and liver. When an individual drinks excessively, the majority of the alcohol is digested in the liver, which increases the overall burden to the liver. Alcohol dehydrogenase $(\mathrm{ADH})$ converts alcohol into acetaldehyde, which is harmful to the body. With the aid of aldehyde dehydrogenase (ALDH), the acetaldehyde is oxidized into acetic acid, which is not harmful to the body, and further catabolized into water and $\mathrm{CO}_{2}$, which are excreted from the body (2).

Long-term alcohol consumption can cause alcoholic liver diseases, including alcoholic hepatitis, alcoholic fatty liver and alcoholic liver cirrhosis (3). According to previous reports, the incidence of alcoholic liver disease has been rising in recent years and alcohol has become the second most common cause of hepatic lesions after hepatitis virus (4). In the USA, 111 million people over 12 years of age drink alcohol, the majority of whom are young people (5). Alcoholic liver disease is a major factor in the incidence of liver disease and overall mortality around the world (6). In the USA, it is estimated that $>2$ million people have alcoholic liver disease (7). Alcoholic cirrhosis is the most common manifestation of alcoholic liver disease and is associated with a greater number of mortalities than all tumors combined (7). Excessive consumption of alcohol causes an accumulation of alcohol in the body, which increases the concentration of alcohol in the brain where it may be up to 10 -fold higher than the serum concentration (7). This accumulation can lead to malfunctioning of the nervous system and cause an excitation impulse that may damage surrounding neurons or be life threatening (8). The anesthetic effect of acetaldehyde can seriously affect, for example, memory, attention, judgment, self-control, eyesight and balance. Long-term drinking can lead to alcohol addiction (alcoholism). When an individual becomes addicted to alcohol, they may experience symptoms such as hand-trembling, confusion, fidgeting and restlessness. Long-term excessive alcohol consumption leads 
to diseases including alcoholic brain disease, brain atrophy and alcoholic dementia (9).

Xingnaojia (XNJ) is a prescription formulated by Professor Guang-Rui Wan of Xinxiang Medical College (Xinxiang, China). By combining the known properties of Chinese herbal medicines, 12 edible traditional Chinese materials were selected, and extracts containing their main functional ingredients were used to create the XNJ formulation. XNJ has been predicted to protect the stomach, liver and brain. This study focuses on the effect of XNJ on the liver function, as well as the learning and memory, of rats with chronic alcoholism, and also explores the mechanism through which XNJ protects the liver and brain.

\section{Materials and methods}

Animals and grouping. Clean male Sprague Dawley rats (weight $140 \pm 20 \mathrm{~g}$ ) were provided by the Animal Center of Zhengzhou University [Zhengzhou, China; License No. scxk (yu) 2005-0001]. The rats were randomly divided into five test groups: A, normal control group; B, model group of alcoholic rats; $\mathrm{C}$, rats given alcohol and a low dose of $\mathrm{XNJ}$; $\mathrm{D}$ rats given alcohol and a high dose of XNJ; and E, positive control group (rats given alcohol and King Drink). Each group comprised 10 rats. The rats were kept at an ambient temperature of $20 \pm 2{ }^{\circ} \mathrm{C}$. Animal manipulations were made according to the Guide to Experimental Animal Treatment (Sept 30, 2006) drafted by the Ministry of Science and Technology of the People's Republic of China. This study was carried out in strict accordance with the recommendations in the Guide for the Care and Use of Laboratory Animals of the National Institutes of Health (8th edition, 2011). The animal use protocol was reviewed and approved by the Institutional Animal Care and Use Committee (IACUC) of Xinxiang Medical University (Xinxiang, China).

Establishment and treatment of rats with chronic alcoholism. XNJ was prepared at Xinxiang Medical College. King Drink tablets (1.0 g per tablet; Batch No. 20101025; China patent, application No. 102631662A) containing primarily puerairin, Hovenia dulcis flavonoids, bamboo leaf flavonoids and gastrodin were provided by Shenzhen Neptunus Group Co., Ltd. (Shenzhen, China). Rats in groups B, C, D and E were fed with an alcohol-water mixture as their only supply of drink. The alcohol concentration was kept at $6 \%$ for 4 weeks and the rats ate freely. Rats in group A ate and drank water freely. During this 4-week period, rats in groups C and D were given XNJ in normal saline $(2 \mathrm{ml} / \mathrm{kg})$ at 9:00 a.m. each day by gavage, where the total flavonoid content of the XNJ was $260 \mathrm{mg}$ and $780 \mathrm{mg}$, respectively; and rats in group E were given normal saline $(2 \mathrm{ml} / \mathrm{mg}$ ) combined with King Drink (1.5 tablets $/ \mathrm{kg}$ ) by gavage. Rats in groups A and B were given the same quantity of normal saline as the other three groups by gavage.

Learning and memory test. This test was carried out following the 4-week period of alcohol/water drinking. Testing was conducted using a Y-maze. The bottom of the instrument container was able to generate electric shocks carrying $0.4 \mathrm{~mA}$ current. Signal lamps were placed at the top of the three arms of the maze. The ability of the rats to avoid electric shocks by following the signal lamps was evaluated. The lights were randomly turned on. Rats were placed in the maze and all lamps were turned on to enable the rats to familiarize themselves with the environment. After $3 \mathrm{~min}$, all the lamps were turned off. One lamp was then turned on, and in the two remaining arms, the electric current was switched on $5 \mathrm{sec}$ later to shock the rats until they ran to the safe area, designated by the signal lamp. Then, all the lamps were turned on for $15 \mathrm{sec}$. Normal reaction was defined as rats reaching the safe area in $10 \mathrm{sec}$ with only one electric shock. Rats that had a normal reaction were described as having passed the learning test, while rats requiring longer reaction times or additional electric shocks to reach the safe area were described as having failed the learning test. If a rat was able to pass $9 / 10$ tests, it was considered to have learned the skill. The number of times that it took for a rat to learn to pass the tests was taken as a quantitative evaluation of its ability to learn and remember spatial details. The fewer attempts a rat required to pass the test, the stronger was its learning ability. All the tests were carried out at night in a quiet environment and under dim light. Rats that were slow to react to the electronic shock were eliminated.

Detection of superoxide dismutase (SOD) in rat brain tissue. Surgical procedures were carried out according to the instructions provided with the SOD detection kit (Nanjing Jiancheng Bioengineering Institute, Nanjing, China). Following the memory test, the rats were anesthetized by an intraperitoneal injection of sodium pentobarbital $(30 \mathrm{mg} / \mathrm{kg})$, then sacrificed by decapitated. The brains were excised immediately and put in an ice bath containing normal saline prior to homogenization. The hydroxylamine oxidation method was used to detect the activity of SOD. One unit of SOD activity was defined as that having an SOD inhibition rate of $50 \%$ for $1 \mathrm{~g}$ tissue protein in $1 \mathrm{ml}$ reaction liquid.

Analysis of glutamate (Glu) levels by high-performance liquid chromatography (HPLC). Glutamic acid standard was bought from Shanghai Kangda Amino Acid Factory (Shanghai, China). Following the memory test and under anesthesia, rats were decapitated and the hippocampus was peeled off and weighed. Then $1 \mathrm{ml}$ methanol-water mixture (1:1) was added to half of the whole hippocampal tissue to prepare a cryogenic homogenate. Following centrifugation $\left(4^{\circ} \mathrm{C}, 10,000 \mathrm{x} \mathrm{g}\right)$ for $15 \mathrm{~min}$, the supernatant was removed and kept at $-80^{\circ} \mathrm{C}$ following membrane filtration for subsequent analysis. Tissue components were measured in units of $\mu \mathrm{g} / \mathrm{g}$. Samples were derived with $o$-phthalaldehyde. HPLC conditions were as follows: ProStar/Dynamax System control system (including Prostar 210 pump, Prostar 363 programmable fluorescence detector, 800 analog-digital converter, ProStar 500 column and temperature box; Agilent Technologies, Santa Clara, CA, USA); mobile phase A, potassium acetate $0.1 \mathrm{M}$; and mobile phase B, carbinol, for processing by bi-gradient elution. The elution procedure was as follows [time $(\mathrm{min})$, percentage B]: $(0,0 \%),(1,5 \%),(10,20 \%),(17,40 \%),(20,60 \%),(22,55 \%)$, $(40,55 \%)$ and $(45,100 \%)$. The mobile phases were filtered using $0.45-\mu \mathrm{m}$ microporous filter membranes, degassed by ultrasound, and run at a flow rate of $1.0 \mathrm{ml} / \mathrm{min}$. The excitation wavelength was $250 \mathrm{~nm}$ and the transmission wavelength 
was $410 \mathrm{~nm}$. The levels of Glu were determined from the peak area.

Analysis of $N$-methyl $D$-aspartate receptor subtype $2 B$ $(N R 2 B)$ by reverse transcription-polymerase chain reaction $(R T-P C R)$. TRIzol reagent and PrimeScript RT-PCR kit were provided by Takara (Dalian) Biotechnology Co., Ltd. (Dalian, China), and primers were synthesized by Sangon Biotech Co., Ltd. (Shanghai, China). SYBR® Premix Ex Taq ${ }^{\mathrm{TM}}$ II kits were purchased from Takara (Dalian) Biotechnology Co., Ltd. Following the memory test, the rats were quickly decapitated. A 30-50-mg sample of brain tissue from the hippocampus was placed on ice and immediately put into TRIzol reagent for extraction of RNA. The RNA was quantified and assessed for purity by measuring the absorbance at $260 \mathrm{~nm}$ and $280 \mathrm{~nm}$. Reverse transcription was performed by following the directions provided with the kit. NR2B primers were generated using Primer 5.0 software (Premier Biosoft, Palo Alto, CA, USA) and their sequences were: Upstream, 5'-CTTACT GAAGGCAATCCTCG-3' and downstream, 5'-TCCTCA GAACACCTTCGCTT-3'. For $\beta$-actin, the primer sequences were: Upstream, 5'-ATGGATGACGATATCGCTGCG-3' and downstream, 5'-TCGTCCCAGTTGGTGACAATG-3'. A PCR mixture was prepared comprising: 10X PCR Buffer II $2.5 \mu \mathrm{l}$, dNTP mixture $1 \mu \mathrm{l}, \mathrm{NR} 2 \mathrm{~B} / \beta$-actin forward primer $(10 \mu \mathrm{mol} / \mathrm{l})$ $1 \mu \mathrm{l}, \mathrm{NR} 2 \mathrm{~B} / \beta$-actin reverse primer $(10 \mu \mathrm{mol} / \mathrm{l}) 1 \mu \mathrm{l}$, Takara Ex Taq HS DNA polymerase $1 \mu \mathrm{l}$, Stencil cDNA $2 \mu \mathrm{l}$ and $\mathrm{ddH}_{2} \mathrm{O}$ $17.5 \mu \mathrm{l}$ (total volume $25 \mu \mathrm{l}$ ). The PCR mixture was placed in PCR reaction tubes and cycled using a M289600 MyCycler PCR instrument (Bio-Rad Laboratories, Inc., Hercules, CA, USA). Amplification conditions were as follows: i) One cycle of denaturing at $94^{\circ} \mathrm{C}$ for $1 \mathrm{~min}$; and ii) 30 cycles of denaturing at $94^{\circ} \mathrm{C}$ for $30 \mathrm{sec}$; annealing at $60^{\circ} \mathrm{C}$ for $30 \mathrm{sec}$; and elongation at $72^{\circ} \mathrm{C}$ for $1 \mathrm{~min}$. PCR results were analyzed using BandScan gel analysis software (Glyko, Hayward, CA, USA) and compared with $\beta$-actin expression in each group. The ratio of the integrated optical density (IOD) of the NR2B gray scale value to the $\beta$-actin reference was analyzed.

Detection of $N R 2 B$ protein expression in rat hippocampus by an immunofluorescence method. The brain tissue in the hippocampus was cut into $20-\mu \mathrm{m}$ sections using a thermostatic freezing microtome and affixed to a polylysine-coated slide. The sample was microwave-repaired for $15 \mathrm{~min}$, and then blocked with goat serum for $20 \mathrm{~min}$. Rabbit anti-mouse NR2B polyclonal antibody (1:100 dilution; Beijing Biosynthesis Biotechnology Co., Ltd., Beijing, China) was added prior to incubation at $4^{\circ} \mathrm{C}$ overnight. Cy3-labeled goat anti-rabbit IgG (1:500; Beijing Biosynthesis Biotechnology Co., Ltd.) was added and the sample was incubated at room temperature for $2 \mathrm{~h}$. Nuclear staining with 4',6-diamidino-2-phenylindole [DAPI; Takara Biotechnology (Dalian) Co., Ltd.] was performed for $3 \mathrm{~min}$. Anti-fluorescence quencher was added to the slide, which was then placed under a BX61 fluorescence microscope (Olympus Corporation, Tokyo, Japan) for observation and imaging.

Serological detection. Abdominal aortic blood was sampled from the rats. The serum was then isolated for the determination of the levels of low-density lipoprotein (LDL), high-density lipoprotein (HDL), triglycerides (TG) and total cholesterol (TCHOL), using a BS-800 automatic biochemical analyzer (Shenzhen Mindray Bio-Medical Electronics Co., Ltd., Shenzhen, China) according to the manufacturer's instructions.

Detection activity of $A D H$ and $A L D H$ in serum and hepatic tissue. Using kits from BioVision Inc. (Milpitas, CA, USA), colorimetry was used to test the activity of ADH and ALDH in serum and hepatic tissue samples from the rats in each group. The experiments were carried out following the instructions provided by the manufacturer.

Detection of cannabinoid receptor 1 (CB1) expression and cyclin-dependent kinase 5 (CDK5) in rat brain hippocampus bywestern blot analysis. The total protein content was extracted from the hippocampal tissue using T-PER (Pierce, Thermo Fisher Scientific, Inc., Rockford, IL, USA) and quantified using a BCA Assay kit (Pik-Day Biotechnology, Haimen, China). The proteins were transferred to a nitrocellulose membrane (Sigma-Aldrich, St. Louis, MO, USA) and then blocked following electrophoresis. Primary anti-CB1 (rabbit anti-rat; 1:300; sc-10066; Santa Cruz Biotechnology, Inc., Dallas, TX, USA) and anti-CDK5 antibodies (rabbit anti-mouse; 1:300; bs-0559R; Beijing Biosynthesis Biotechnology Co., Ltd., Beijing, China) overnight at $4^{\circ} \mathrm{C}$. Membranes were washed three times for 5 min in PBS, then secondary goat anti-rabbit IgG Cy3-labeled antibodies (1:300; A-0521; Beijing Biosynthesis Biotechnology, Co., Ltd.) were added for hybridization for $2 \mathrm{~h}$. Electrochemiluminescence analysis was conducted using SuperSignal ${ }^{\circledR}$ West Dura Extended Duration Substrate (Pierce, Thermo Fisher Scientific, Inc.). The optical density of the film was measured using a gel-scanner, and $\beta$-actin was used as the internal reference. The IOD was calculated to determine the relative amount of test protein. The experiment was repeated three times, and the mean was calculated.

Statistical analysis. Results are shown as the mean \pm standard deviation. SPSS software package, version 12.0 (SPSS, Inc., Chicago, IL, USA) was used to conduct the statistical analysis. Comparisons among groups were performed using one-way analysis of variance and least significant difference detection.

\section{Results}

Effect of XNJ on the learning and memory of rats with chronic alcoholism. According to the Y-maze behavior test, the learning ability and memory of rats with chronic alcoholism was significantly weakened compared with that of normal control rats $(\mathrm{P}<0.01)$. However, high and low doses of XNJ had a protective effect on rats with chronic alcoholism (Table I).

Effect of XNJ on SOD activity in brain homogenate. the activity of SOD in the brain homogenate of the model group was significantly reduced compared with that in the control group $(\mathrm{P}<0.01)$. In the groups treated with a high or low dose of XNJ, the activity of SOD in the brain homogenate increased significantly $(\mathrm{P}<0.05$ and $\mathrm{P}<0.01$, respectively; Table II).

Effect of XNJ on NR2B mRNA expression. Image analysis results following RT-PCR demonstrated that the expression 
Table I. Effect of XNJ formulation on learning and memory.

\begin{tabular}{lc}
\hline Group & $\begin{array}{c}\text { No. of training times } \\
\text { for an accurate response }\end{array}$ \\
\hline Control & $37.38 \pm 11.61$ \\
Model & $104.88 \pm 12.98^{\mathrm{a}}$ \\
Positive control & $97.00 \pm 7.71$ \\
Low-dose & $93.25 \pm 7.63^{\mathrm{b}}$ \\
High-dose & $46.00 \pm 5.78^{\mathrm{c}}$ \\
\hline
\end{tabular}

Results are from a Y-maze test. If the rat escaped to the safe area directly within $10 \mathrm{sec}$, it was considered a correct response; otherwise, it was considered an incorrect response. Nine correct responses per 10 trials indicated that the rat had learnt to escape. Training times taken in learning are a measure the performance of spatial learning and memory. One way analysis of variance and least significant difference multiple-range tests were used to determine the differences of means. ${ }^{a} \mathrm{P}<0.01$ vs. control; ${ }^{b} \mathrm{P}<0.05,{ }^{\mathrm{c}} \mathrm{P}<0.01$ vs. model. Data are expressed as mean \pm standard deviation ( $\mathrm{n}=10$ rats per group; experiment repeated three times). XNJ, Xingnaojia.

Table II. Effect of XNJ formulation on brain levels of SOD (x10 $10^{3} \mathrm{U} /$ gprot).

\begin{tabular}{lc}
\hline Group & SOD level \\
\hline Control & $26.9000 \pm 2.1647$ \\
Model & $19.2800 \pm 1.7645^{\mathrm{a}}$ \\
Positive control & $19.4167 \pm 1.5295$ \\
Low-dose & $21.3512 \pm 1.5545^{\mathrm{b}}$ \\
High-dose & $25.2756 \pm 1.7510^{\mathrm{c}}$ \\
\hline
\end{tabular}

${ }^{\mathrm{a}} \mathrm{P}<0.01$ vs. control; ${ }^{\mathrm{b}} \mathrm{P}<0.05,{ }^{\mathrm{c}} \mathrm{P}<0.01$ vs. model. All data are expressed as mean \pm standard deviation $(n=10$ rats per group; experiment repeated three times). One way analysis of variance and least significant difference multiple-range test were used to determine the differences of means. XNJ, Xingnaojia; SOD, superoxide dismutase.

level of NR2B mRNA was highest in the model group, and lowest in the control group. In the groups treated with high or low doses of XNJ, the NR2B mRNA levels were intermediate between those of the control and model groups, but were notably lower than the levels in the model group $(\mathrm{P}<0.01$; Table III, Fig. 1).

Effect of XNJ on NR2B protein expression in the hippocampus of rats with chronic alcoholism. The expression of NR2B protein in the hippocampus is shown in Fig. 2. NR2B-positive cell cytoplasm is red. In the model group, compared with the control group, there was a large number of fluorescent stained cells, which were densely distributed. The fluorescence intensity was increased in the rats with chronic alcoholism. The administration of XNJ reduced the number of NR2B protein-positive cells. The number of NR2B protein-positive cells was reduced significantly as the dose of $\mathrm{XNJ}$ was increased, in comparison with the number in the model group.
Table III. Comparison of NR2B mRNA levels (gray values).

\begin{tabular}{lc}
\hline Group & NR2B mRNA \\
\hline Control & $0.62 \pm 0.05$ \\
Model & $1.27 \pm 0.06^{\mathrm{a}}$ \\
Positive control & $1.23 \pm 0.09$ \\
Low-dose & $1.08 \pm 0.07^{\mathrm{b}}$ \\
High-dose & $0.83 \pm 0.08^{\mathrm{b}}$ \\
\hline
\end{tabular}

${ }^{\mathrm{a}} \mathrm{P}<0.01$ vs. control; ${ }^{\mathrm{b}} \mathrm{P}<0.01$ vs. model. Gray scale value analysis of NR2B mRNA bands. mRNA was reverse-transcribed and amplified with $\beta$-actin as the internal control. All data are expressed as mean \pm standard deviation $(n=10$ rats per group; experiment repeated three times). One way analysis of variance and least significant difference multiple-range test were used to determine the differences of means. NR2B, N-methyl-D-aspartate receptor subtype 2B.

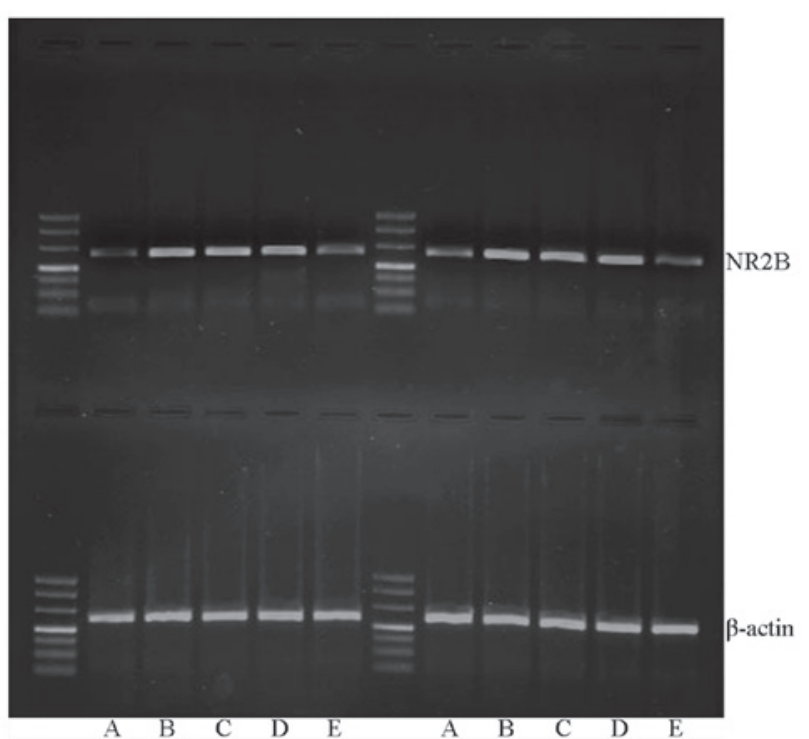

Figure 1. Expression of NR2B mRNA. Lane A, control; lane B, model; lane $\mathrm{C}$, positive control; lane $\mathrm{D}$, low-dose $\mathrm{XNJ}$; lane $\mathrm{E}$, high-dose $\mathrm{XNJ}$. NR2B, N-methyl D-aspartate receptor subtype 2B; XNJ, Xingnaojia.

Effect of XNJ on Glu levels in the hippocampus of rats with alcoholism. HPLC data have previously shown that the treatment of chronic alcoholism can reduce the Glu level in the rat hippocampus. The results of the present study reveal that high or low doses of XNJ had no effect on the levels of Glu in the hippocampal tissue of rats with alcoholism (Table IV, Fig. 3).

Effect of XNJ on the expression of $C B 1$ in the hippocampus of rats with chronic alcoholism. As shown in Fig. 4, the expression level of CB1 in the hippocampal tissue of rats in the model group increased significantly compared with that in the control group, whereas CB1 expression was markedly reduced in rats treated with high and low doses of XNJ compared with that in the model group.

Effect of XNJ on the expression of CDK5 in the hippocampus of rats with chronic alcoholism. As shown in Fig. 4, the expression level of CDK5 was increased in the rats with 
A

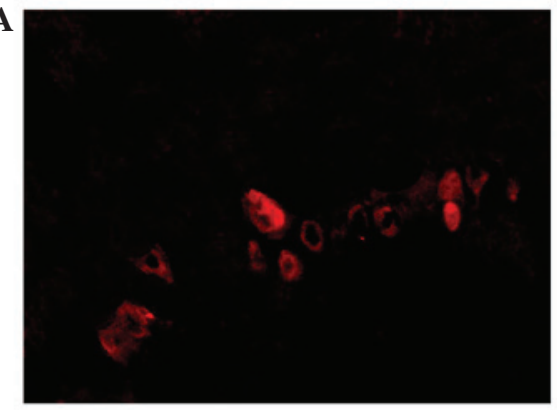

D

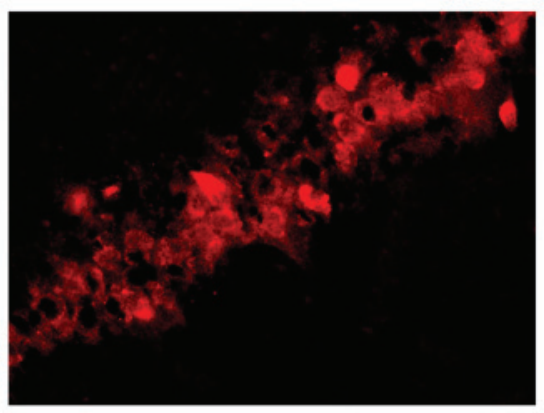

G

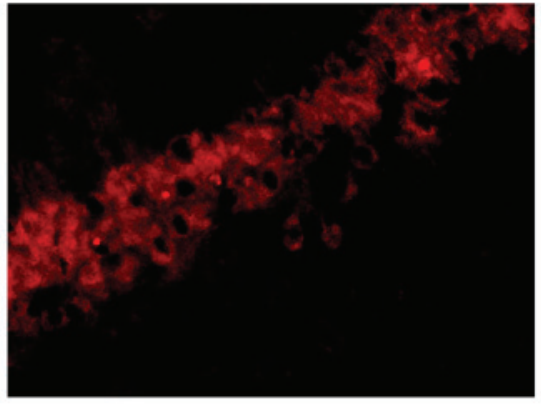

$\mathbf{J}$

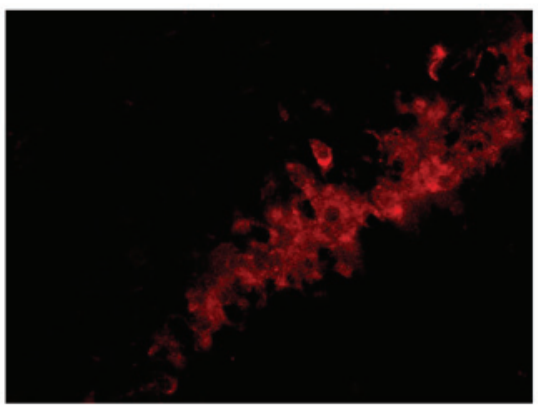

$\mathbf{M}$

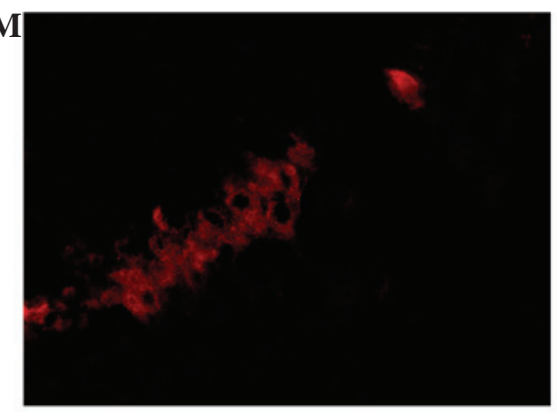

$\mathbf{B}$

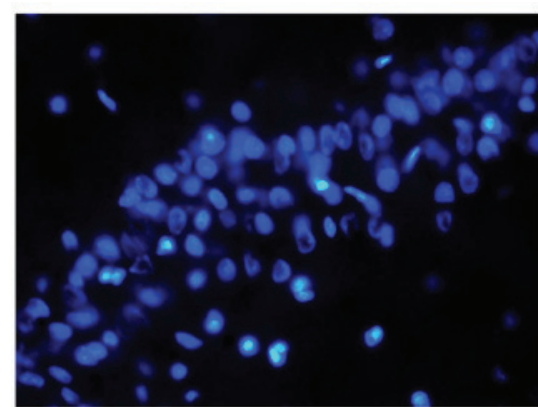

E
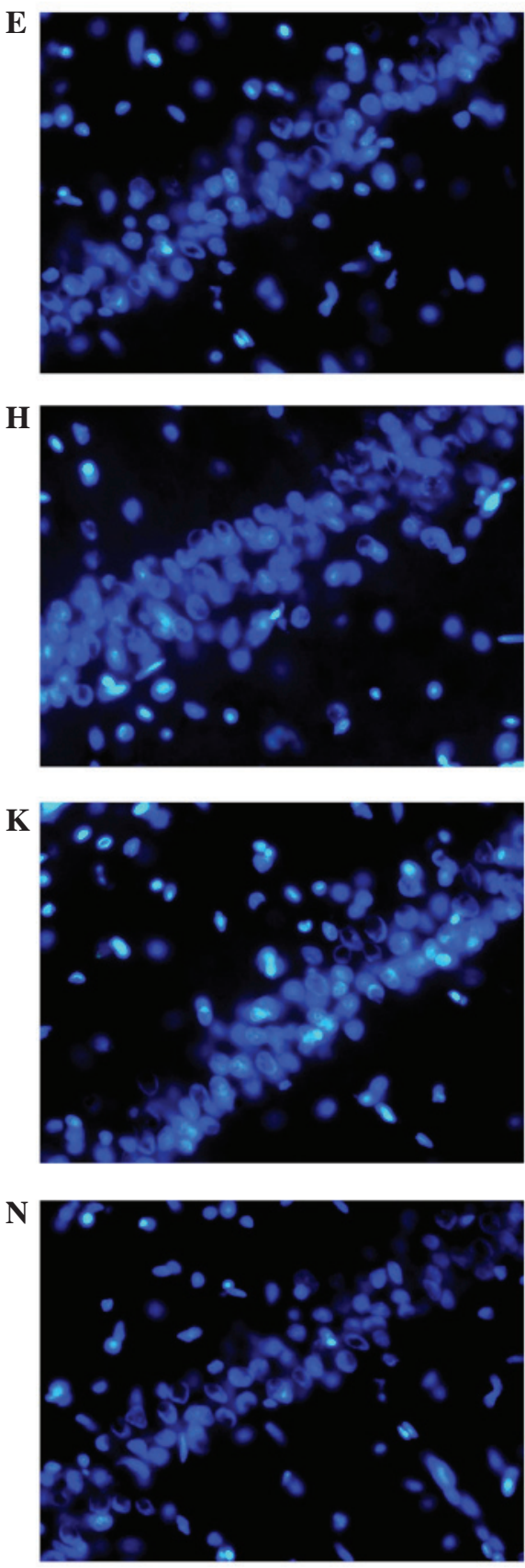

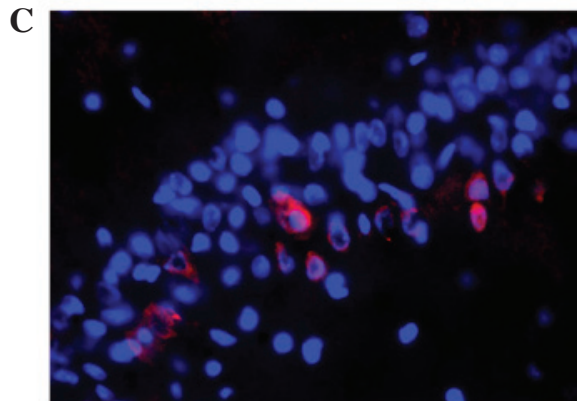

F
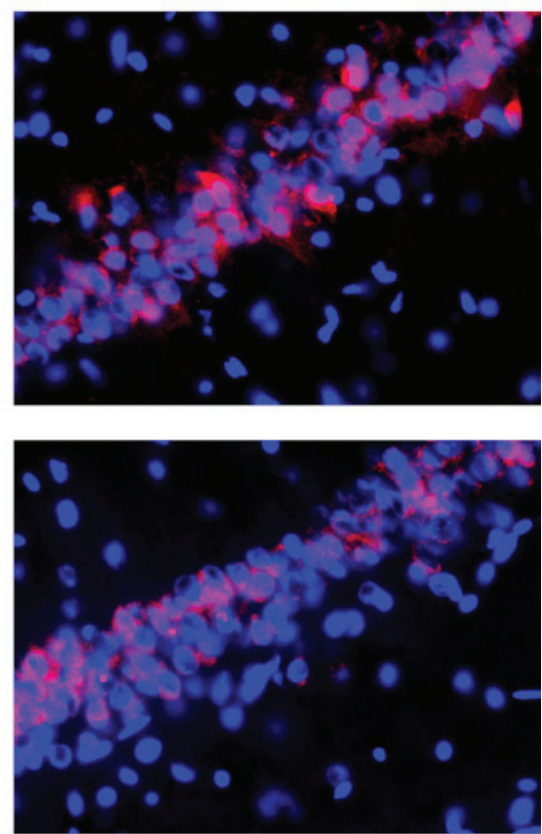

$\mathbf{L}$

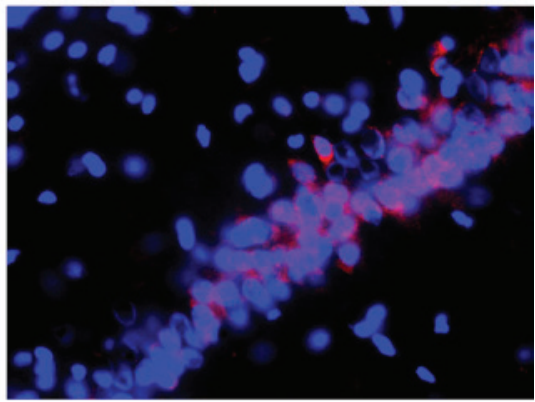

$\mathbf{O}$

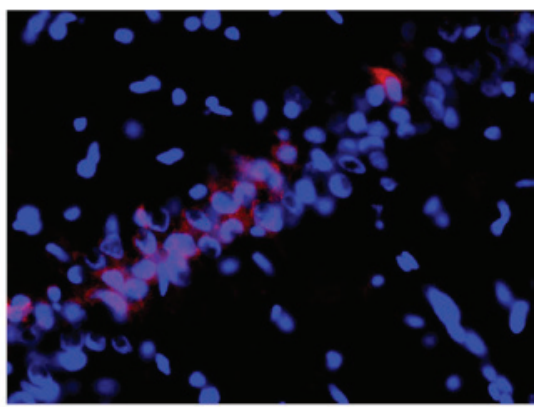

Figure 2. Representative photomicrographs of NR2B (Cy3-labeled, red fluorescence; DAPI-labeled, blue fluorescence; magnification, x400) with immunofluorescent staining in the rat hippocampus. A large number of neurons were fluorescently stained in the hippocampus in the rats from the model group, with a dense distribution and increased fluorescence intensity in comparison with the control group. By contrast, the number and density of fluorescently stained neurons and the fluorescence intensity were decreased in the rats from the drug-treated groups compared with those in the model group. (A) Positive NR2B expression, (B) DAPI staining and (C) merged image of staining in the normal group. (D) Positive NR2B expression, (E) DAPI staining and (F) merged image of staining in the model group; (G) Positive NR2B expression, (H) DAPI staining and (I) merged image of staining in the positive control group. (J) Positive NR2B expression, (K) DAPI staining and (L) merged image of staining in the group treated with a low dose of XNJ. (M) Positive NR2B expression, (N) DAPI staining and $(\mathrm{O})$ merged image of staining in the group treated with a high dose of XNJ. NR2B, N-methyl D-aspartate receptor subtype 2B; DAPI, 4',6-diamidino-2-phenylindole; XNJ, Xingnaojia. 

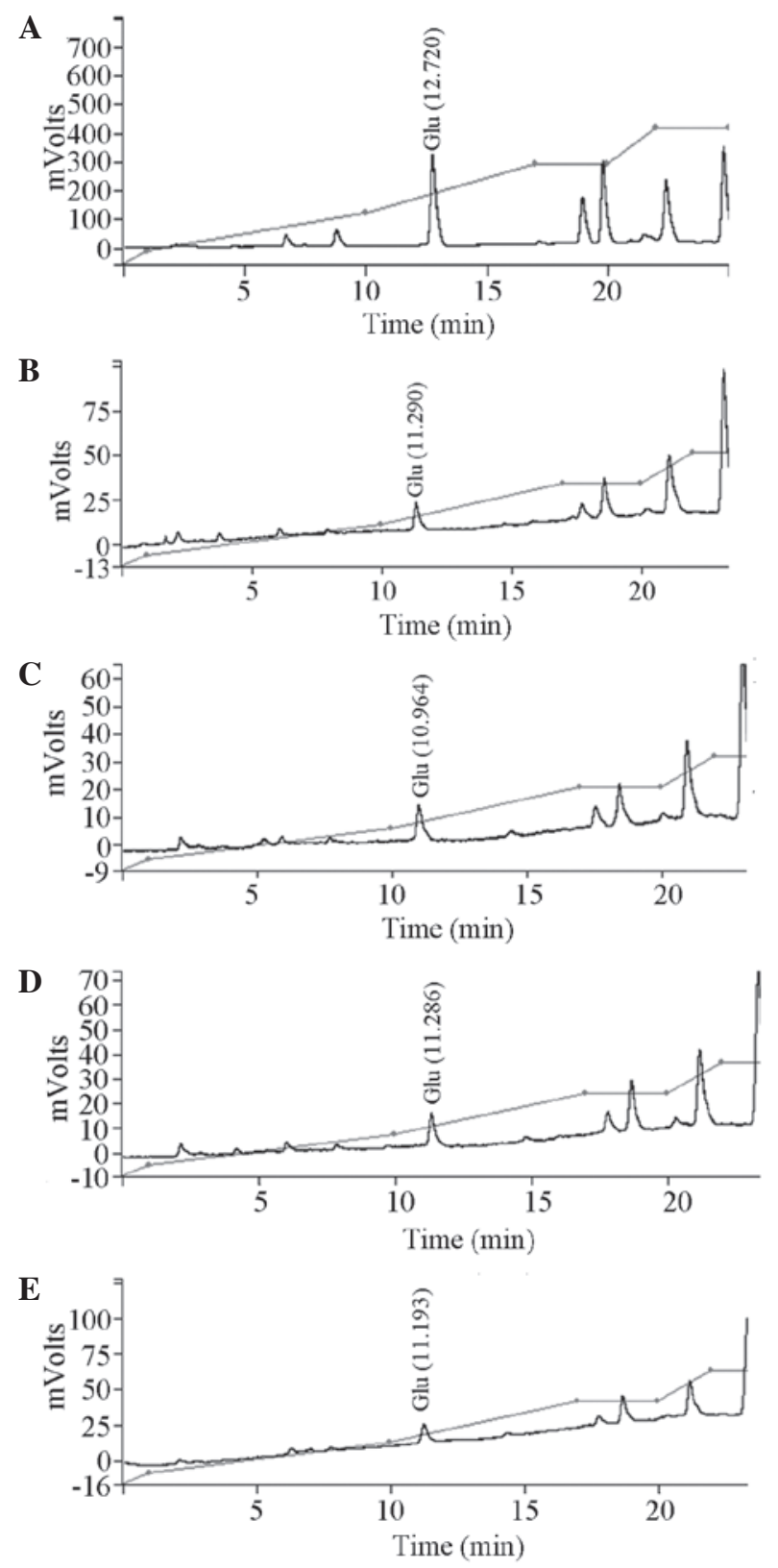

Figure 3. Results of HPLC analysis of glutamate (Glu) levels in the rat hippocampus. (A) Control, (B) model, (C) positive control, (D) low-dose XNJ and (E) high-dose XNJ groups. HPLC, high-performance liquid chromatography; XNJ, Xingnaojia.

chronic alcoholism compared with that in the control group. The expression level of CDK5 was reduced in the groups receiving a high or low dose of $\mathrm{XNJ}$ compared with that in the model group.

Hepatocyte ultrastructure alteration. In group A (the control group), rounded or oval-shaped nuclei were observed in the hepatocytes and the cell membranes retained their integrity. Clear nucleoli were also observed, in addition to clear and complete nuclear membranes. Furthermore, the appearance of the mitochondria was normal and the cristae structure could be distinctly seen. Occasionally, tiny lipid droplets were observed in the cells (Fig. 5A). In group B (model group with alcoholism), it was observed that certain cells had an incomplete membrane and marginalized heterochromatin in the nucleus.
Table IV. Effect of XNJ formulation on brain levels of glutamate in rats with chronical alcoholism $(\mu \mathrm{mol})$.

\begin{tabular}{lc} 
Group & Glutamate level \\
\hline Control & $3.49 \pm 0.70$ \\
Model & $0.24 \pm 0.06^{\mathrm{a}}$ \\
Positive control & $0.27 \pm 0.07^{\mathrm{a}}$ \\
Low-dose & $0.29 \pm 0.07^{\mathrm{a}}$ \\
High-dose & $0.28 \pm 0.06^{\mathrm{a}}$ \\
\hline
\end{tabular}

${ }^{\mathrm{a}} \mathrm{P}<0.01$ vs. control. All data are expressed as mean \pm standard deviation ( $n=10$ rats per group; experiment repeated three times). One way analysis of variance and least significant difference multiple-range test were used to determine the differences of means. XNJ, Xingnaojia.

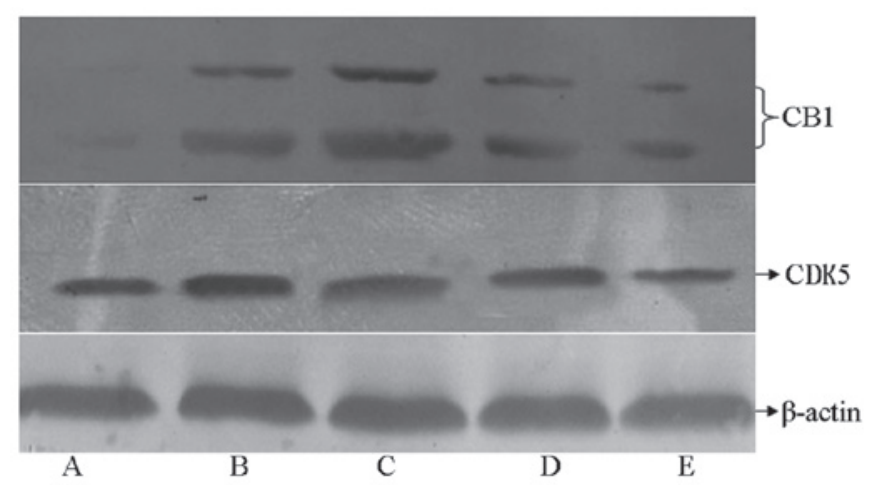

Figure 4. Expression of CB1 and CDK5 in the rat hippocampus as demonstrated by western blotting. Lane A, control; lane B, model; lane C, positive control; lane D, low-dose XNJ; lane E, high-dose XNJ. CB1, cannabinoid receptor 1; CDK5, cyclin-dependent kinase 5; XNJ, Xingnaojia.

Mitochondria were swollen in these hepatocytes and their cristae were segmented and blurred. There were a number of lipid droplets of various sizes in the cytoplasm (Fig. 5B). In group $\mathrm{C}$ (the positive control group), there were small amounts of lipid droplets in the hepatocytes and a normal number of mitochondria (Fig. 5C). Group D (low-dose XNJ group) exhibited lipid droplets of various sizes in the hepatic cells (Fig. 5D). In group E (high-dose XNJ group), the number of mitochondria in hepatocytes was increased and lipid droplets were seldom observed (Fig. 5E).

Effect of XNJ on levels of LDL, HDL, TG and TCHOL in the serum of rats with alcoholism. The levels of LDL, TG and TCHOL in the serum increased and those of HDL decreased significantly in the model group compared with those in the control group $(\mathrm{P}<0.05)$ and these changes were significantly attenuated in the group treated with XNJ when compared with those in the model group $(\mathrm{P}<0.05$; Tables $\mathrm{V}$ and $\mathrm{VI})$.

Effect of XNJ on the activity of ADH in the serum and liver tissue of rats with alcoholism. Tests of ADH activity in the serum and liver tissue of rats in each group were conducted using a colorimetric assay. As the results show, the activity of $\mathrm{ADH}$ increased significantly in the model group and the two 

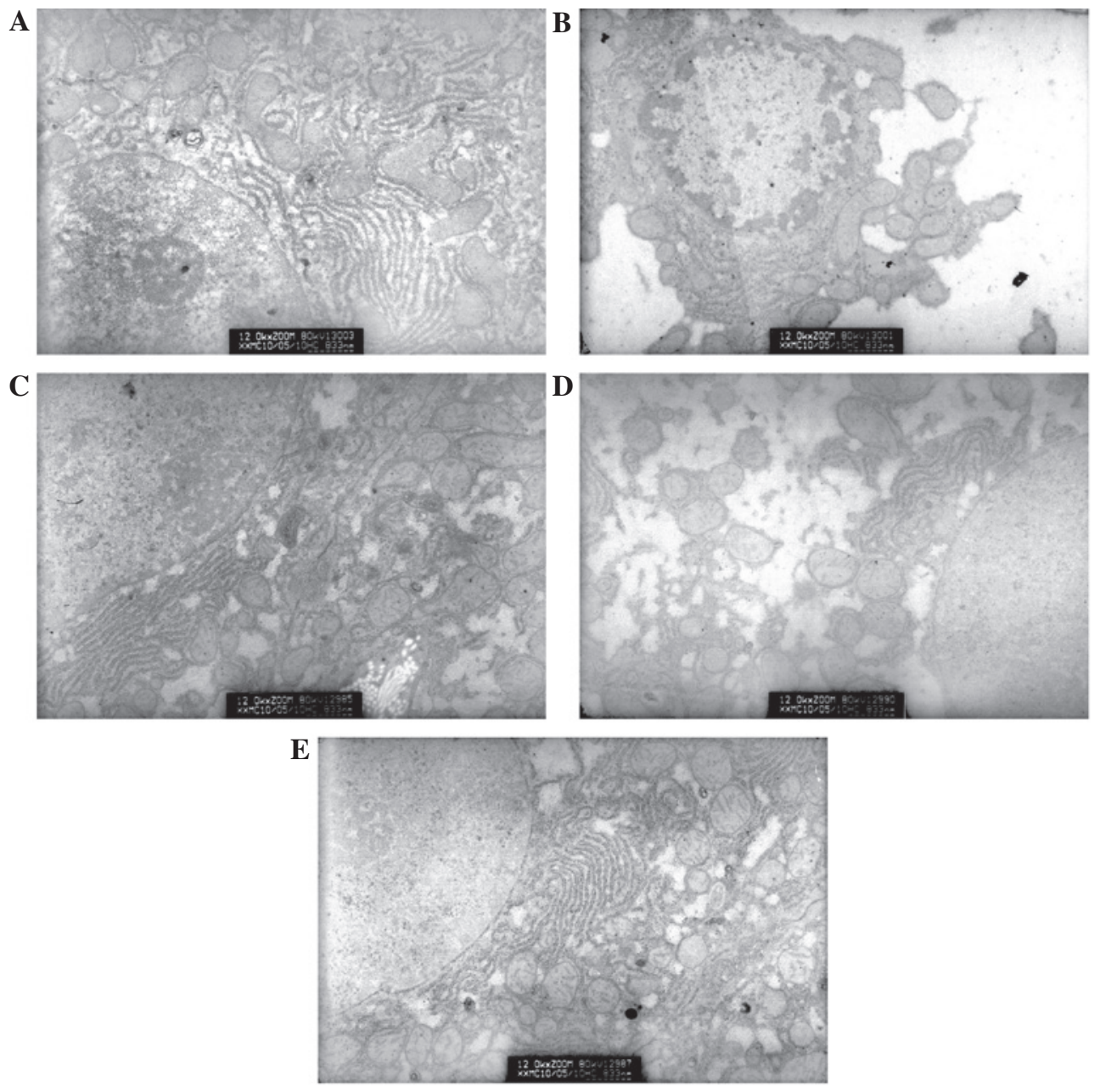

Figure 5. Changes in the ultrastructure of rat liver cells in each group. (A) Normal group, (B) model group with alcoholism, (C) positive control group, (D) low-dose XNJ and (E) high-dose XNJ groups. XNJ, Xingnaojia.

groups receiving $\mathrm{XNJ}$ treatment, all of which differed from the control group $(\mathrm{P}<0.05)$. However, there was no significant difference in the activity of ADH among groups $\mathrm{B}, \mathrm{C}, \mathrm{D}$ and $\mathrm{E}$ (Table VII).

Effect of XNJ on the activity of ALDH in the serum and liver tissue of rats with alcoholism. The activity of ALDH in the rat serum and liver tissue was detected by colorimetry. According to the results, the activity of ALDH in the model group and group receiving low-dose $\mathrm{CNJ}$ exhibited no significant differences compared with that in the control group. However, the ALDH activity was markedly increased in the high dose XNJ group, and was significantly different from that detected in the model group $(\mathrm{P}<0.05$; Table VIII).

\section{Discussion}

Based on the properties and function of traditional Chinese medicines and knowledge of modern pharmacology, 12 traditional Chinese medicines were selected and their primary functional ingredients were extracted to create the $\mathrm{XNJ}$ formulation. The essential ingredients include isoflavones from kudzu root and raisin tree seeds, as well as flavones from bamboo leaves, Gastrodia tuber and resistant starch. Previous studies have demonstrated that XNJ can significantly improve learning and memory in humans, alleviate neurological damage and protect the liver $(10,11)$. In order to verify the neutralizing effect of XNJ on the physiological effects of alcoholic drinks, King Drink, a sobering preparation that has been applied clinically for many years, was used as a positive control.

The major damaging effect of alcoholism on the nervous system is a toxic effect on neurons and damaging effects on learning and memory (12). The results of the present study demonstrate that learning and memory in the model rat group was much worse than that of the control group $(\mathrm{P}<0.01)$, whereas learning and memory was improved in the groups receiving high- or low-dose $\mathrm{XNJ}(\mathrm{P}<0.05$ or $\mathrm{P}<0.01)$. There was no apparent distinction between the positive control and model groups. This indicates that the XNJ formulation may have a protective effect against the neurological damage caused by chronic alcoholism in rats.

In order to investigate the mechanism by which $\mathrm{XNJ}$ protects the nervous system, the activity of SOD and the levels of NR2B, Glu, CDK5 and CB1 in rat brain tissue were assayed. The experimental results reveal that the SOD activity of rats in the model group was significantly reduced when compared with that in the control group, while SOD activity increased markedly in the XNJ-treated groups compared with those in the model group. This shows that the activity of the antioxi- 
Table V. Effect of XNJ on LDL and HDL in the serum of rats with alcoholism (mmol/l).

\begin{tabular}{llc}
\hline Group & LDL & HDL \\
\hline Control & $2.29 \pm 0.14$ & $0.54 \pm 0.16$ \\
Model & $2.89 \pm 0.17^{\mathrm{a}}$ & $0.23 \pm 0.04^{\mathrm{a}}$ \\
Positive control & $2.59 \pm 0.12^{\mathrm{b}}$ & $0.52 \pm 0.07^{\mathrm{b}}$ \\
Low-dose & $2.46 \pm 0.21^{\mathrm{b}}$ & $0.46 \pm 0.15^{\mathrm{b}}$ \\
High-dose & $2.57 \pm 0.07^{\mathrm{b}}$ & $0.40 \pm 0.08^{\mathrm{b}}$ \\
\hline
\end{tabular}

${ }^{\mathrm{a}} \mathrm{P}<0.05$ vs. control; ${ }^{\mathrm{b}} \mathrm{P}<0.05$ vs. model. All data are expressed as mean \pm standard deviation ( $\mathrm{n}=10$ rats per group; experiment repeated three times). One way analysis of variance and least significant difference multiple-range test were used to determine the differences of means. LDL, low-density lipoprotein; HDL, high-density lipoprotein; XNJ, Xingnaojia.

Table VI. Effect of XNJ on TCHOL and TG in the serum of rats with alcoholism $(\mathrm{mmol} / \mathrm{l})$.

\begin{tabular}{llc}
\hline Group & TCHOL & TG \\
\hline Control & $1.72 \pm 0.08$ & $0.71 \pm 0.16$ \\
Model & $2.04 \pm 0.20^{\mathrm{a}}$ & $1.00 \pm 0.11^{\mathrm{a}}$ \\
Positive control & $1.75 \pm 0.50^{\mathrm{b}}$ & $0.69 \pm 0.06^{\mathrm{b}}$ \\
Low-dose & $1.75 \pm 0.12^{\mathrm{b}}$ & $0.79 \pm 0.10^{\mathrm{b}}$ \\
High-dose & $1.77 \pm 0.24^{\mathrm{b}}$ & $0.76 \pm 0.08^{\mathrm{b}}$ \\
\hline
\end{tabular}

${ }^{\mathrm{a}} \mathrm{P}<0.05$ vs. control; ${ }^{\mathrm{b}} \mathrm{P}<0.05$ vs. model. All data are expressed as mean \pm standard deviation $(n=10$ rats per group; experiment repeated three times). One way analysis of variance and least significant difference multiple-range test were used to determine the differences of means. TCHOL, total cholesterol; TG, triglycerides; XNJ, Xingnaojia.

Table VII. Effect of XNJ on the activity of ADH in the serum and hepatic tissue of rats with alcoholism $(\mathrm{mU} / \mathrm{ml})$.

\begin{tabular}{lcc}
\hline Group & Serum & Liver \\
\hline Control & $66,855.05 \pm 865.16$ & $153,667.41 \pm 736.55^{\mathrm{a}}$ \\
Model & $166,160.25 \pm 1495.30^{\mathrm{a}}$ & $292,414.59 \pm 1063.95$ \\
Positive control & $164,725.47 \pm 1309.36$ & $307,917.94 \pm 1777.64$ \\
Low-dose & $167,004.97 \pm 1099.24$ & $296,895.20 \pm 1100.82$ \\
High-dose & $164,899.20 \pm 1334.29$ & $283,956.57 \pm 1111.98$
\end{tabular}

${ }^{\mathrm{a}} \mathrm{P}<0.05$ vs. control. All data are expressed as the mean \pm standard deviation ( $n=10$ rats per group; experiment repeated three times). One way analysis of variance and least significant difference multiple-range test were used to determine the differences of means. $\mathrm{ADH}$, alcohol dehydrogenase; XNJ, Xingnaojia.

dant enzyme SOD in the brain tissue was increased with the use of XNJ, which in turn suggests that that XNJ may help to neutralize free oxygen radicals and mitigate their damaging
Table VIII. Effect of XNJ on the activity of ALDH in the serum and hepatic tissue of rats with alcoholism (U/ul).

\begin{tabular}{lcc}
\hline Group & Serum & Liver \\
\hline Control & $0.0719 \pm 0.0094$ & $0.5920 \pm 0.0173$ \\
Model & $0.0732 \pm 0.0105$ & $0.6272 \pm 0.0171$ \\
Positive control & $0.0747 \pm 0.0143$ & $0.6107 \pm 0.0461$ \\
Low-dose & $0.0738 \pm 0.0160$ & $0.6276 \pm 0.0591$ \\
High-dose & $0.1626 \pm 0.0097^{\mathrm{a}}$ & $0.9224 \pm 0.0861^{\mathrm{a}}$
\end{tabular}

${ }^{\mathrm{a}} \mathrm{P}<0.05$ vs. model. All data are expressed as mean \pm standard deviation ( $\mathrm{n}=10$ rats per group; experiment repeated three times). One way analysis of variance and least significant difference multiple-range test were used to determine the differences of means. ALDH, aldehyde dehydrogenase; XNJ, Xingnaojia.

effects on brain tissue. Increasing the activity of antioxidant enzymes may be a mechanism by which XNJ protects the brain from the effects of alcohol. The main ingredients of XNJ are isoflavones and flavones, which have lipid peroxidation and free radical-mitigating effects.

The hippocampus is an important region for neuron plasticity and is closely associated with learning ability and memory in mammals (13). However, NMDA receptors, which are the main regulators of synaptic plasticity and long-term potentiation (LTD), are richly expressed in brain tissue (14). Studies have shown that LTD in the hippocampus is mediated by Glu and NMDA receptors (15). The NMDA receptor NR2B plays an important role in neural plasticity (16). A previous study demonstrated that alcohol is able to affect the NR2B receptor, leading to neuronal damage and changes in learning and memory (17). The present study has shown that with high-dose XNJ administration, the expression of NR2B mRNA in the hippocampus of rats with chronic alcoholism was much lower than that in the model group $(\mathrm{P}<0.01)$. This data suggests that the XNJ formulation has a protective effect against brain damage in rats with chronic alcoholism and that this effect is probably due to regulation of the expression of NR2B protein. However, the molecular mechanism of this protective effect is unknown.

Researchers have shown that the excessive activation of NMDA receptors increases the concentration of $\mathrm{Ca}^{2+}$ continuously, which leads to $\mathrm{Ca}^{2+}$ overload and can activate a series of enzymes related to cytotoxicity, such as protein kinase $\mathrm{C}$ (PKC) and nitric oxide synthase (NOS). These enzymes can damage the main components of the cellular lipid membrane and cytoskeletal proteins of nerve cells and cause gradual necrosis of neurons (18). Vanillin and $p$-hydroxybenzaldehyde, which are components of Gastrodia tuber, have been shown to significantly suppress the Glu-stimulated intracellular increase of $\mathrm{Ca}^{2+}$ in IMR-32 neuroblastoma cells and apoptosis (19). Gastrodin can also suppress the levels of $\mathrm{Ca}^{2+}$ in PC12 cells stimulated by Glu, which suggests that the calcium channel could be the target of gastrodin components that suppress the effects of excitotoxicity (19). It is hypothesized that XNJ downregulates NR2B receptors and suppresses $\mathrm{Ca}^{2+}$ mobilization, which in turn protects against neurological damage in rats 
with chronic alcoholism. In this manner, XNJ may improve rat learning and memory. After assaying the levels of Glu in the rat hippocampus, it was found that there was no significant change in Glu levels in the rats receiving XNJ. This indicates that the function of XNJ is not associated with Glu regulation.

In order to determine the specific mechanism of this process, two factors that are closely connected with learning, memory and neurological damage, namely CB1 and CDK5, were examined. Humans have used marijuana throughout history. Cannabinoids are the active ingredients in marijuana and act on CB1 in the brain to bring feelings of euphoria and reward. CB1 is mostly expressed in the central nervous system (CNS) and belongs to the class of G-protein coupled receptors. $\mathrm{CB} 1$ is closely associated with neurogenesis, neural development, synapse formation, learning and memory, food intake and energy metabolism $(20,21)$. CB1 is the most abundant receptor in mammalian brains. By binding to its cognate ligand, CB1 transfers signals between neurons and regulates a wide range of signaling mechanisms. Previous studies have shown that there is a close correlation between CB1 and the neurotoxic effects of alcoholism (21). In the CNS, endogenous cannabinoids function as reverse neurotransmitters following their release from post-synaptic neurons and act on the pre-synaptic membranes of neurons bearing CB1. When CB1 receptors are activated and coupled with the voltage-dependent $\mathrm{Ca}^{2+}$ channel, the channel closes and the influx of $\mathrm{Ca}^{2+}$ is reduced. Through this mechanism, neurotransmitters such as $\gamma$-aminobutyric acid (GABA) and Glu are released in lesser amounts in the presynaptic membrane of neurons (22).

CDK5 is a member of the cyclin-dependent kinase family with a molecular weight of $35 \mathrm{kD}$. It is a proline-directed serine/threonine protein kinase that phosphorylates a serine/threonine residue with a $\mathrm{C}$-terminally adjacent proline residue. CDK5 is abundantly expressed in the nervous system and is regulated by the activator proteins P35 and P39 (23). A previous study has shown that CDK5 is involved in synaptic plasticity in the nervous system as well as learning ability and memory (24). Moreover, it is implicated in drug addiction. Earlier studies have shown that P35 and P39, activator proteins of CDK5, are regulated by intracellular $\mathrm{Ca}^{2+}$ and hydrolyzed to P25 and P29. P35, P39, P25 and P29 can form a heterodimer with, and thereby activate CDK5; the half-lives of P25 and P29 are long, which would result in the excessive activation of CDK5, thereby causing neurotoxicity, such as neuronal apoptosis and cytoskeleton damage (24). It has been reported that certain components of traditional Chinese medicines can function as calcium channel blockers that are able to suppress the overactivation of CDK5 and protect the brain from the damage caused by calcium overload in neurons to a certain degree (25). The results of the present study demonstrate that XNJ clearly reduced the expression of CB1 and CDK5 in brain tissue. Based these results, it is considered that the effects of $\mathrm{XNJ}$ on the calcium signaling pathway require further investigation.

In order to investigate the protective effect of XNJ on the liver and the brain, the ultrastructure of hepatic tissue in rats with chronic alcoholism was observed under an electron microscope. In addition, the activity of ADH and ALDH in rat serum and hepatic tissue, as well as the levels of LDL, HDL, TG and in serum were measured. The effects of XNJ were found to include significant improvement of the damaged ultrastructure of the hepatic tissue in rats with chronic alcoholism, and reductions of the levels of LDL, TCHOL and TG in the serum of rats with chronic alcoholism accompanied by an increase in the level of HDL. This indicates that XNJ is able to regulate the lipid metabolic disorder caused by alcohol.

ADH and ALDH together constitute the oxidative pathway by which alcohol is metabolized into acetic acid in the liver (26). ADH is a crucial enzyme that enables liver cells to metabolize short-chain alcohols and is responsible for oxidizing alcohol into acetaldehyde. ALDH is located in the mitochondria of liver cells and is the enzyme responsible for metabolizing acetaldehyde into acetic acid, which is harmless to the body. Chronic alcoholism can lead to increased activity of ADH but not ALDH, which leads to a buildup of acetaldehyde and chronic acetaldehyde intoxication with time. Rats with chronic alcoholism are very similar to humans with this condition; the quantity of $\mathrm{ADH}$ is abundant and similar to that found in the normal human body. However, there is a significant distinction among individuals and their levels of ALDH. A significant proportion of people lack ALDH. As a result, in these individuals, alcohol is metabolized into acetaldehyde by $\mathrm{ADH}$, but cannot be converted to acetic acid by ALDH. This leads to the accumulation of acetaldehyde in the body, which in turn causes symptoms of drunkenness, including sickness, inarticulation, staggering and unconsciousness (27). Certain individuals have ALDH with reduced enzymatic activity (27); therefore, if they drink too much or too quickly, at a rate beyond the enzymatic activity of ALDH, they can become inebriated. XNJ can significantly increase the activity of ALDH in rats with chronic alcoholism and accelerate the breakdown of acetaldehyde into $\mathrm{H}_{2} \mathrm{O}$ and $\mathrm{CO}_{2}$, thereby protecting the liver.

In conclusion, XNJ was able to significantly neutralize the adverse effects of alcohol, improve memory, alleviate neural injuries and protect liver function. The formulation comprises natural herbs, providing wide application prospects. Although the mechanisms underlying the protective effects are discussed in the present study, the exact molecular mechanism and pathway remain unclear and require further study.

\section{Acknowledgements}

This study was supported by Major Research Projects of Department of Science and Technology of Henan Province of China (No: 121100910300).

\section{References}

1. De Rick A, Vanheule S and Verhaeghe P: Alcohol addiction and the attachment system: an empirical study of attachment style, alexithymia, and psychiatric disorders in alcoholic inpatients. Subst Use Misuse 44: 99-114, 2009.

2. Whitfield JB: ADH and ALDH genotypes in relation to alcohol metabolic rate and sensitivity. Alcohol Alcohol Suppl 2: 59-65, 1994.

3. Bruha R, Dvorak K and Petrtyl J: Alcoholic liver disease. World J Hepatol 4: 81-90, 2012.

4. Diehl AM: Liver disease in alcohol abusers: clinical perspective. Alcohol 27: 7-11, 2002.

5. Gao B and Bataller R: Alcoholic liver disease: Pathogenesis and new therapeutic targets. Gastroenterology 141: 1572-1585, 2011.

6. Williams R: The pervading influence of alcoholic liver disease in hepatology. Alcohol Alcohol 43: 393-397, 2008. 
7. Barve A, Khan R, Marsano L, Ravindra KV and McClain C: Treatment of alcoholic liver disease. Ann Hepatol 7: 5-15, 2008.

8. Dickov A, Vuckovic N, Martinovic-Mitrovic S, et al: Disorder verbal memory in alcoholics after delirium tremens. Eur Rev Med Pharmacol Sci 16: 1052-1060, 2012.

9. Chopra K and Tiwari V: Alcoholic neuropathy: possible mechanisms and future treatment possibilities. Br J Clin Pharmacol 73: 348-362, 2012.

10. Li S, Wan J, Chen WJ and Wan GR: Effect of Xinnaojia formula on learning and memory and expression of NR2B in the hippocampus of rats with chronic alcoholism. Zhong Guo Ying Yong Sheng Li Xue Za Zhi 27: 5-6, 2011 (In Chinese).

11. Du AL, Li S, Wan J, Wang D, Zhu F, Meng L and Wan GR: Effect of Xinnaojia formula on the liver damage of rats with chronic alcoholism. Zhong Guo Lao Nian Xue Za Zhi 35: 156-157, 2015 (In Chinese)

12. Kruman II, Henderson GI and Bergeson SE: DNA damage and neurotoxicity of chronic alcohol abuse. Exp Biol Med (Maywood) 237: 740-747, 2012.

13. Burke CJ, Tobler PN, Baddeley M and Schultz W: Neural mechanisms of observational learning. Proc Natl Acad Sci USA 107: 14431-14436, 2010.

14. Stoneham ET, Sanders EM, Sanyal M and Dumas TC: Rules of engagement: Factors that regulate activity-dependent synaptic plasticity during neural network development. Biol Bull 219. 81-99, 2010.

15. Li R, Huang FS, Abbas AK and Wigström H: Role of NMDA receptor subtypes in different forms of NMDA-dependent synaptic plasticity. BMC Neurosci 8: 55, 2007.

16. Mallon AP, Auberson YP and Stone TW: Selective subunit antagonists suggest an inhibitory relationship between NR2B and NR2A-subunit containing N-methyl-D-aspartate receptors in hippocampal slices. Exp Brain Res 162: 374-383, 2005.

17. Kash TL, Matthews RT and Winder DG: Alcohol inhibits NR2B-containing NMDA receptors in the ventral bed nucleus of the stria terminalis. Neuropsychopharmacology 33: 1379-1390, 2008.
18. Xin WK, Kwan CL, Zhao XH, Xu J, Ellen RP, McCulloch CA and Yu XM: A functional interaction of sodium and calcium in the regulation of NMDA receptor activity by remote NMDA receptors. J Neurosci 25: 139-148, 2005.

19. Lee YS, Ha JH, Yong CS, Lee DU, Huh K, Kang YS, Lee SH, Jung MW and Jim JA: Inhibitory effects of constituents of Gastrodia elata Bl. on glutamate-induced apoptosis in IMR-32 human neuroblastoma cells. Arch Pharm Res 22: 404-409, 1999.

20. Howlett AC, Barth F, Bonner TI, Cabral G Casellas P, Devane WA, Felder CC, Herkenham M, Mackie K, Martin BR, et al: International union of pharmacology. XXVII. Classification of cannabinoid receptors. Pharmacol Rev 54: 161-202, 2002.

21. Khasabova IA, Khasabov S, Paz J, Harding-Rose C, Simone DA and Seybold VS: Cannabinoid type-1 receptor reduces pain and neurotoxicity produced by chemotherapy. J Neurosci 32 : 7091-7101, 2012.

22. Maccioni P, Colombo G and Carai MA: Blockade of the cannabinoid CB1 receptor and alcohol dependence: Preclinical evidence and preliminary clinical data. CNS Neurol Disord Drug Targets 9: 55-59, 2010

23. Polissidis A, Galanopoulos A, Naxakis G, Papahatjis D, Papadopoulou-Daifoti Z and Antoniou K: The cannabinoid CB1 receptor biphasically modulates motor activity and regulates dopamine and glutamate release region dependently. Int J Neuropsychopharmacol 16: 393-403, 2013.

24. Benavides DR and Bibb JA: Role of CDK5 in drug abuse and plasticity. Ann NY Acad Sci 1025: 335-344, 2004

25. Lee MS, Kwon YT, Li M, Peng J, Friedlander RM and Tsai LH: Neurotoxicity induced cleavage of p35 to p25 by calpain. Nature 405: 360-364, 2000.

26. Jelski W and Szmitkowski M: Alcohol dehydrogenase (ADH) and aldehyde dehydrogenase (ALDH) in the cancer diseases. Clin Chim Acta 395: 1-5, 2008.

27. Ehlers CL, Liang T and Gizer IR: ADH and ALDH polymorphisms and alcohol dependence in Mexican and native Americans. Am J Drug Alcohol Abuse 38: 389-394, 2012. 